\title{
Advances in Bulk Metallic Glasses
}

\author{
Peter K. Liaw, Gongyao Wang, and Judy Schneider
}

Bulk metallic glasses (BMG) are relatively new amorphous materials with excellent properties and promising applications. A collection of articles regarding bulk metallic glasses, which presents very good discussions on some important issues and the latest developments, have been accepted for publication in $J O M$. Four papers were published in February 2010 JOM, and three additional papers complete the topic in April.

First, "The Fracture Toughness of Bulk Metallic Glasses" by Jian Xu, Upadrasta Ramamurty, and Evan Ma reviews and summarizes the current understanding of the mechanics and micromechanisms of BMG toughness and highlights the needs for future research.

Next, "Thin Film Metallic Glasses: Preparations, Properties, and Applications" by J.P. Chu et al. reviews and reports important and interesting results regarding the glass-forming ability, thermal, and mechanical properties of thin-film metallic glasses as well as potential applications.

Finally, "Bending-fatigue Behavior of Bulk Metallic Glasses and Their Composites" by Gongyao Wang and Peter K. Liaw summarizes and compares the three- and four-point-bending-fatigue characteristics of BMGs and their composites in different load- ing conditions and environments. The factors affecting the fatigue behavior of BMGs and their composites are discussed, and the mechanisms of fatigue-crack initiation and propagation in BMGs are addressed in this paper.

The papers presented in February were:

"Understanding the Properties and Structure of Metallic Glasses at the Atomic Level" by T. Egami ${ }^{1}$ reviewed the difficulties inherent to the problem regarding the behavior of glasses and liquids at the atomic level and the microscopic mechanisms that control the properties of metallic glasses and some recent advances.

"Mechanical Response of Metallic Glasses: Insights from In-situ High Energy X-ray Diffraction" by Mihai Stoica et $\mathrm{al}^{2}$ reviewed the use of the synchrotron radiation for evaluating the elastic-plastic response of BMGs. Experimental results from the available literature were compared and analyzed.

"Amorphous Metals for Hard-Tissue Prosthesis" by Marios D. Demetriou et $\mathrm{al}^{3}$ described features of the performance of amorphous alloys relevant to the hard-tissue prosthesis and proposed guidelines for the development of new biocompatible amorphous alloys suitable for hard-tissue prosthesis.
"Metallic Glasses: Gaining Plasticity for Microsystems" by Yong Yang et $\mathrm{al}^{4}{ }^{4}$ summarized the experimental findings related to size effects in metallic glasses. The enhanced plasticity of metallic glasses in small volumes, together with their high mechanical strengths and remarkable thermoplastic formability, strongly implies that metallic glasses are the promising materials for fabricating the next generation of micro- and nano-devices.

These BMG papers in two JOM issues comprehensively discuss the fundamental mechanisms as well as possible applications regarding metallic glasses. We hope these papers are very helpful to our BMG community. Besides the fundamental research on BMGs, it is critically important to study the science and engineering aspects of BMGs applications.

\section{References}

1. T. Egami, JOM, 62 (2) (2010), p. 70.

2. Mihai Stoica et al., Ref. 1, p. 76

3. Marios D. Demetriou et al., Ref. 1, p. 83

4. Yong Yang et al., Ref. 1, p. 93.

Peter K. Liaw, professor, and Gongyao Wang, research associate, are with the Department of Materials Science and Engineering, The University of Tennessee, Knoxville, TN 37996, USA; Judy Schneider, associate professor, is with the Mechanical Engineering Department, Mississippi State University, MS 39762, USA, and is the JOM advisor from the Mechanical Behavior of Materials Committee of the TMS Structural Materials Division. 